\title{
Association of Anticholinergic Load With Impairment of Complex Attention and Memory in Schizophrenia
}

\author{
Michael J. Minzenberg, M.D. \\ John H. Poole, Ph.D. \\ Cynthia Benton, M.D.
}

Sophia Vinogradov, M.D.

\begin{abstract}
Objective: The authors' goals were 1) to establish a clinically useful standard index of the relative anticholinergic potency of psychiatric medications; 2 ) to determine which cognitive functions are most affected by the administration of anticholinergic medications to patients with schizophrenia; and 3) to compare in vitro and clinically derived indexes of anticholinergic load in predicting these cognitive impairments.
\end{abstract}

\begin{abstract}
Method: One hundred six clinically stable patients with schizophrenia were given a brief neuropsychological battery and evaluated on a standard symptom rating scale. The anticholinergic load associated with their psychiatric medications was estimated by using 1) a pharmacological index, calculated from a compilation of published studies reporting in vitro brain muscarinic receptor antagonism, and 2) a clinical index, based on clinician ratings of the anticholinergic side effects of medications. The authors analyzed the correlations of both indexes with the neuropsychological measures and with summary neuropsychological factor scores.
\end{abstract}

Results: The clinical and pharmacological anticholinergic indexes were highly correlated with each other and showed virtually identical associations with neuropsychological measures. Anticholinergic load was associated with lower scores on measures of attention and declarative memory, including several measures of auditory and visual memory and two tests of complex attention, but was unrelated to intelligence, simple attention, working memory, executive functions, conceptual fluency, or motor speed.

Conclusions: This pattern of cognitive impairment with central cholinergic antagonism is consistent with emerging models of the functional anatomy of ascending forebrain cholinergic subsystems. Both pharmacological and clinical indexes show utility in predicting the effects of anticholinergic load on cognition in schizophrenia. Doses of psychiatric medication within the range of routine pharmacotherapy practice may have clinically significant effects on memory and complex attention in patients with schizophrenia; these effects may contribute as much as one-third to two-thirds of the memory deficit typically seen in patients with schizophrenia.
$\mathrm{M}$ phrenia spectrum disorders, including antipsychotics, antidepressants, and antiparkinsonian medications, have clinically significant anticholinergic effects. The general role of such medications in iatrogenic memory impairment and other cognitive deficits in schizophrenia has been recognized (1). Nevertheless, the precise nature of the cognitive impairment resulting from the use of these medications in this clinical population is not well characterized. This is a crucial unresolved issue in the clinical practice of psychopharmacology, given the association between cognitive impairment and functional status in schizophrenia $(2,3)$. By the same token, the effect of anticholinergic medications on cognition is a likely confounding factor that is often ignored in the laboratory research on neurocognition in clinical populations treated with these medications.

The few reported empirical studies addressing anticholinergic effects on cognition in schizophrenia have gener- ally found isolated impairments in declarative memory (4-11). However, these studies have generally used small sample sizes and limited neurocognitive batteries. In addition, these studies have been inspired by an older, obsolete model of mammalian forebrain cholinergic functional anatomy. In this earlier model, the entire basal forebrain cholinergic system was postulated specifically to subserve memory functions in an undifferentiated manner. In contrast, advances in basic neuroscience methodology have revealed the role of cholinergic subsystems in mediating distinct cognitive functions (see references 12 and 13 for reviews).

For example, the ascending cholinergic cortical projection from the nucleus basalis of Meynert is now recognized as playing a central role in attentional function, particularly in tasks of divided, cross-modal, or sustained attention (14-17). The different roles of muscarinic and nicotinic acetylcholine receptors have not yet been clarified. These two receptor types, both found in terminal 
fields of mammalian cholinergic projection neurons, differ in virtually every important aspect, such as brain distribution, chemical structure, the set of ligands that bind them, and the cellular effects associated with activation of these receptors (18). Nevertheless, among the currently available medications indicated for psychiatric disorders, none is recognized as having significant activity at the nicotinic receptor. In contrast, many medications have recognized antimuscarinic activity. Thus, the primary issue of clinical significance is to evaluate the role of antimuscarinic effects on cognition (we use the term "anticholinergic" to refer to antimuscarinic data in the remainder of the paper because of the familiarity of this term).

It is also unclear which method is more appropriate in the clinical setting for estimating the anticholinergic load conferred by psychiatric medications. Pharmacological indexes, established from in vitro acetylcholine receptor binding studies, are often used to calculate the anticholinergic load that individuals are exposed to either in clinical settings or in studies of normal human cognition. In vitro estimates of anticholinergic load do not account for pharmacokinetic factors, which may account for significant variation in the bioavailability of these medications. Although a laboratory assay has been developed to determine the anticholinergic load from human serum samples (19), this method remains costly and unavailable in most clinical and research settings. In addition, the relationship between serum concentrations of these compounds and brain synaptic biophase concentrations (i.e., the rate of brain penetration) is unknown for most medications at this time. Therefore, the need remains for an inexpensive, easy, and reliable method for estimating clinically relevant anticholinergic loads.

We performed the present analysis on the clinical and neuropsychological data from our established database in order to address the following questions: 1) Which cognitive functions are most affected by the administration of anticholinergic medications to schizophrenic patients? 2) How do in vitro and clinically derived indexes of anticholinergic load compare in predicting these cognitive impairments? 3) What is the magnitude of the cognitive effects that might result from a change in anticholinergic medication doses?

To address these questions, we calculated mean in vitro muscarinic receptor binding affinity from all published reports that provided binding data on any medications currently prescribed in the United States for the treatment of psychiatric disorders. We believe that this report is the first to comprehensively integrate muscarinic receptor binding data from the literature in order to establish a standard index of relative anticholinergic potency of psychotropic medications. We also calculated a clinical index of anticholinergic load based on clinicians' ratings of the relative potency of these medications in inducing common peripheral anticholinergic side effects.

\section{Method}

\section{Subjects}

All subjects $(\mathrm{N}=106)$ were outpatients recruited from the San Francisco VA Medical Center and the surrounding community and diagnosed with either schizophrenia or schizoaffective disorder according to the Structured Clinical Interview for DSM-IV Axis I Disorders. These subjects were recruited to participate in other concurrent studies of neurocognitive impairment in schizophrenia. Exclusion criteria for all subjects were history of head trauma, psychoactive substance abuse within 6 months before study participation, or English as a second language. After complete description of the study to the subjects, written informed consent was obtained.

Eighty-one (76\%) of the patients were men; their mean age was 39.9 years $(\mathrm{SD}=11.3)$; their mean education level was 13.7 years $(\mathrm{SD}=2.3$ ); their mean illness duration (from time of first symptoms) was 20 years ( $\mathrm{SD}=10.7)$; and their mean Global Assessment of Functioning Scale (GAF) (DSM-IV, p. 32) score was 50 (SD=9.0). All patients were clinically stable, with no change for at least 1 month in their current medication regimen prescribed by their outpatient physician. Treatment adherence was assessed by interview at each testing session.

Reference values on cognitive tests were obtained from 50 healthy comparison subjects ( 29 male, 21 female) who were recruited from hospital employees and contacts in the community, excluding candidates with any current axis I diagnosis or suspected schizophrenia spectrum disorders in first-degree relatives (based on a brief structured interview). The group of patients with schizophrenia and the comparison group were matched for age (for patients, mean=39.9, $\mathrm{SD}=11.3$, and for comparison subjects, mean=39.4, $\mathrm{SD}=12.6$ ), parental education (for patients, mean= 13.7 years, $\mathrm{SD}=3.4$, and for comparison subjects, mean=13.6, $\mathrm{SD}=$ 3.5), and parental occupation level (Hollingshead index) (for patients, mean $=5.3, \mathrm{SD}=2.1$, and for comparison subjects mean $=5.0$, $\mathrm{SD}=2.1$ ). There were $18 \%$ more males among the patients with schizophrenia $(76 \%$ of the patients were men, compared with $58 \%$ of the comparison subjects) ( $\mathrm{p}<0.10$, Fisher's exact test). Patients with schizophrenia completed fewer years of education (mean=13.7, $\mathrm{SD}=2.3$ ) than comparison subjects (mean=15.5, $\mathrm{SD}=$ 2.2) $(\mathrm{t}=4.9, \mathrm{df}=151, \mathrm{p}<0.001)$, as is expected for appropriately matched groups of patients with schizophrenia and comparison subjects (20).

\section{Medications}

Eighty-six percent of the patients with schizophrenia or schizoaffective disorder $(\mathrm{N}=91)$ were receiving neuroleptics; their mean chlorpromazine equivalent dose was $520 \mathrm{mg} /$ day. Table 1 provides a list of all psychiatric medications prescribed to these subjects that have anticholinergic activity. Nonpsychiatric medications currently prescribed to each patient were recorded but not entered into the subsequent analysis (Table 1). None of these nonpsychiatric medications is considered to have significant anticholinergic activity.

\section{Clinical and Neuropsychological Assessment}

To evaluate current symptom severity, we administered an extended version of the Positive and Negative Symptom Scale (21), which included 10 supplementary items from the Comprehensive Assessment of Symptoms and History (22), to all of the patients. In addition, all patients were assessed with a neuropsychological battery that included portions of the WAIS-R (23), the Trail Making Test (Trails A and B) (24), the Digit Span and Visual Span from the Wechsler Memory Scale-Revised (WMS-R) (25), the Wisconsin Card Sorting Test (26), the Stroop Color and Word Test, Victoria version (27), the Rey-Osterrieth Complex Figure Design (with copy and recall after 20 minutes according to the Denman 
TABLE 1. Relative Anticholinergic Potency of Psychiatric Medications $^{\text {a }}$

\begin{tabular}{|c|c|c|c|c|c|}
\hline \multirow[b]{2}{*}{ Medication } & \multicolumn{2}{|c|}{$\begin{array}{c}\text { Pharmacological } \\
\text { Index }\end{array}$} & \multicolumn{2}{|c|}{$\begin{array}{l}\text { Clinical } \\
\text { Index }\end{array}$} & \multirow{2}{*}{$\begin{array}{l}\text { Number of } \\
\text { Subjects } \\
\text { Receiving } \\
\text { Medication }^{d}\end{array}$} \\
\hline & Mean & SD & Mean & SD & \\
\hline Amitriptyline $\mathrm{e}^{\mathrm{e}}$ & 10 & 2.2 & 47 & 87 & 1 \\
\hline Amoxapine & 733 & & 113 & 131 & 1 \\
\hline Benztropine & 1 & & 1 & & 26 \\
\hline Biperiden & 0.7 & & $-^{f}$ & & \\
\hline Chlorpromazine ${ }^{\mathrm{e}}$ & 47 & 34 & 78 & 138 & 4 \\
\hline Clozapine ${ }^{g}$ & 8 & 65 & 85 & 140 & 8 \\
\hline Desmethylimipramine $^{\mathrm{e}}$ & 105 & 31 & 93 & 117 & \\
\hline Diphenhydramine & 147 & & 30 & 120 & 11 \\
\hline Doxepin $^{\mathrm{e}}$ & 41 & 13 & 50 & 92 & 2 \\
\hline Fluphenazine & $-^{f}$ & & 13 & 8 & 11 \\
\hline Haloperidol & $-^{f}$ & & 13 & 8 & 15 \\
\hline Imipramine $\mathrm{e}^{\mathrm{e}}$ & 51 & 11 & 69 & 146 & 1 \\
\hline Loxapine & 293 & & $-^{f}$ & & \\
\hline Mesoridazine & 47 & & $-^{f}$ & & \\
\hline Nortriptyline $\mathrm{e}^{\mathrm{e}}$ & 73 & 26 & 85 & 115 & 4 \\
\hline Olanzapine & 17 & & 19 & 34 & 9 \\
\hline Paroxetine & 73 & & 67 & 85 & 6 \\
\hline Perphenazine & 1470 & & 14 & 14 & 15 \\
\hline Pimozide & 490 & & $-^{f}$ & & \\
\hline Prochlorperazine & 367 & & $-^{f}$ & & \\
\hline Promazine & 98 & & $-^{f}$ & & \\
\hline Quetiapine & 733 & & 228 & 231 & 4 \\
\hline Risperidone & $-f$ & & 11 & 6 & 24 \\
\hline Sertraline & 490 & & $-^{f}$ & & 8 \\
\hline Thioridazine & 12 & & 61 & 111 & 4 \\
\hline Thiothixene & $-^{f}$ & & 15 & 13 & 8 \\
\hline Trifluoperazine & 490 & & 17 & 16 & 10 \\
\hline Trihexyphenidyl & 1.6 & & 2 & 5 & 6 \\
\hline
\end{tabular}

a Medications are included only if in vitro data were available on anticholinergic activity in mammalian brain preparations and the medications were prescribed for psychiatric indications. Data are expressed as milligram amount equivalent in anticholinergic activity to $1 \mathrm{mg}$ of benztropine mesylate and standard deviation. Nonpsychiatric medications prescribed to the 106 patients in our database at the time of study included cimetidine $(\mathrm{N}=1)$, enalapril $(\mathrm{N}=$ 1), hydrochlorothiazide $(\mathrm{N}=1)$, hydroxyurea $(\mathrm{N}=1)$, Premarin $(\mathrm{N}=1)$, propranolol $(\mathrm{N}=2)$, and propothyrouracil $(\mathrm{N}=1)$. See text for derivation of anticholinergic indexes.

${ }^{\mathrm{b}}$ Unless noted, for the pharmacological index, only one or two published reports contributing data to the index were available. Standard deviations of the mean are not defined for data points based on fewer than three studies. Standard deviation is undefined for benztropine because it was the reference value.

' Based on ratings of medication's effects on their patients by 10 psychiatrists with experience in clinical psychopharmacology.

${ }^{d}$ Number of subjects in our database prescribed the medication at the time of study; total exceeds 106 because many patients were prescribed more than one medication concurrently.

e Three published reports contributed data to the index.

${ }^{f}$ Anticholinergic activity is effectively zero (either in vitro data or as judged by the clinical panel) or otherwise not available.

$\mathrm{g}$ Five published reports contributed data to the index.

scoring system) (28), the California Verbal Learning Test (29), facial memory from the Test of Memory and Learning (30), the Serial Visuospatial Learning Test (a test of visual position learning and recall analogous to the California Verbal Learning Test, under development in our laboratory), the Controlled Oral Word Association Test (31), the Ruff Figural Fluency Test (32), and the Finger Tapping Test (33). All symptom ratings and neurocognitive assessments were performed within a $2-4$-week period.

\section{Derivation of Anticholinergic Indexes}

Pharmacological index. A MEDLINE search of the existing literature was first conducted with the keywords "anticholinergic," "acetylcholine," "neuropsychology," and "cognition." These keywords were used to capture all reports addressing the effects of anticholinergic medications on neurocognitive or neuropsychological function. All English-language reports of in vitro acetylcholine receptor binding studies using brain tissue and any of the psychotropic medications either currently prescribed to our subjects or commonly prescribed in general psychiatric practice were included (34-41). These reports included both human and rodent brain tissue preparations because many of the newer medications do not have reported receptor binding affinities from human tissue. Relative affinities for brain muscarinic receptors (across all subtypes) were typically reported as $K_{d}$ values for displacement of 3-quinuclidinyl benzilate binding in brain homogenates. For medications that had been assayed in multiple studies, a mean pharmacological anticholinergic potency was calculated. These data were then converted to relative "benztropine equivalents" on the basis of the $K_{d}$ for benztropine mesylate reported by Snyder and Yamamura (41) in order to derive values for in vitro activity that could be directly compared with those derived from a clinical reference. The total "pharmacological" anticholinergic load (expressed relative to $1 \mathrm{mg}$ benztropine mesylate) was then calculated for each subject (Table 1).

Clinical index. We recruited a panel of 10 practicing psychiatrists with extensive experience in clinical psychopharmacology. This panel was composed of staff psychiatrists from the Department of Veterans Affairs Medical Center, San Francisco, and Langley Porter Psychiatric Institute at the University of California, San Francisco. The median length of experience in active clinical psychopharmacological experience among these raters was 20 years; in addition, eight of these raters have published empirical research in the field of pharmacology. The clinicians were asked to rate the clinical potency of medications, relative to $1 \mathrm{mg}$ of benztropine mesylate p.o., on the basis of their patients' complaints of dry mouth, blurred vision, and constipation, the most prevalent nonpsychotropic side effects of anticholinergic medications. Each clinician completed these ratings independently, without being informed of one another's evaluations. Based on these ratings, mean anticholinergic potency was calculated for each medication (Table 1), and the total "clinical" anticholinergic load was calculated for each patient, also in benztropine equivalents.

\section{Data Analysis}

Using the age of each of our 106 patients and published agestratified norms, we calculated age-adjusted $\mathrm{z}$ scores for each patient's neuropsychological test data (positive z scores indicate above-average performance). Several measures that were not normally distributed (clinical benztropine mesylate equivalents, Trails A and B, and all Wisconsin Card Sorting Test and Stroop Color and Word Test variables) were successfully normalized by log transformation before correlation analyses. The pharmacological benztropine mesylate equivalent variable was normalized by an inverse transformation before analysis (the sign was reversed on inversion to maintain the direction of the correlations). This permitted use of parametric procedures for all statistical tests. Student's t test was used to compare the cognitive test scores of the patients with schizophrenia and those of the control subjects. Pearson's $r$ was used to evaluate the correlations of the two anticholinergic indexes with one another and with cognitive scores. Fisher's r-z transformation, adjusted for nonindependence of measures (42), was used to test whether the two anticholinergic indexes had significantly different patterns of correlation with the cognitive test scores.

The normalizing data transformations noted above did not change the pattern of significant findings (as assessed by nonparametric Mann-Whitney U and Spearman's rho tests, not reported here). Because all neuropsychological test scores were transformed to z scores, all associations of anticholinergic load with neuropsychological impairment are expressed as negative 
MINZENBERG, POOLE, BENTON, ET AL.

TABLE 2. Performance on Neuropsychological Tests of 106 Patients With Schizophrenia or Schizoaffective Disorder and 50 Healthy Comparison Subjects ${ }^{\mathrm{a}}$

\begin{tabular}{|c|c|c|c|c|c|c|c|}
\hline \multirow[b]{2}{*}{ Neuropsychological Measure } & \multicolumn{2}{|c|}{ Comparison Subjects } & \multicolumn{2}{|c|}{ Patients } & \multicolumn{3}{|c|}{ Analysis } \\
\hline & Mean & SD & Mean & SD & $\mathrm{t}$ & df & $\mathrm{p}$ \\
\hline General intelligence: full-scale IQ (WAIS-R) & 112 & 13 & 97 & 12 & 6.22 & 135 & $<0.0005$ \\
\hline \multicolumn{8}{|l|}{ Simple attention } \\
\hline Digit Span forward (WMS-R) & 0.21 & 1.21 & -0.59 & 1.17 & 3.53 & 135 & 0.001 \\
\hline Visual Span forward (WMS-R) & 0.00 & 0.91 & -0.24 & 1.07 & 1.18 & 130 & 0.24 \\
\hline Visuomotor search (Trails A) & 0.08 & 0.53 & -0.82 & 1.16 & 6.18 & 129 & $<0.0005$ \\
\hline \multicolumn{8}{|l|}{ Complex attention } \\
\hline Divided attention (Trails B) & 0.22 & 0.48 & -0.81 & 1.23 & 7.00 & 134 & $<0.0005$ \\
\hline Response Control (Stroop Color and Word Test, color-word) & 0.40 & 1.03 & -1.00 & 1.97 & 4.50 & 116 & $<0.0005$ \\
\hline Copy accuracy (Rey-Osterrieth Complex Figure Design) & 0.39 & 0.85 & -0.29 & 1.55 & 3.21 & 109 & 0.002 \\
\hline \multicolumn{8}{|l|}{ Short-term memory } \\
\hline \multicolumn{8}{|l|}{ Verbal learning and recall } \\
\hline Initial recall (California Verbal Learning Test, trial 1) & 0.24 & 1.12 & -0.89 & 1.07 & 5.30 & 133 & $<0.0005$ \\
\hline Serial recall (California Verbal Learning Test, sum of trials $1-5$ ) & 0.51 & 0.92 & -1.19 & 1.25 & 8.51 & 80 & $<0.0005$ \\
\hline \multicolumn{8}{|l|}{ Visuospatial learning and recall } \\
\hline Initial recall (Serial Visuospatial Learning Test, trial 1) & 0.06 & 1.00 & -0.45 & 1.08 & 2.40 & 125 & 0.02 \\
\hline Serial recall (Serial Visuospatial Learning Test, sum of trials 1-5) & 0.23 & 0.93 & -0.60 & 1.05 & 4.09 & 125 & $<0.0005$ \\
\hline Delayed recall (Rey-Osterrieth Complex Figure Design) & 0.09 & 0.99 & -0.81 & 1.03 & 4.50 & 132 & $<0.0005$ \\
\hline \multicolumn{8}{|l|}{ Facial learning and recall (Test of Memory and Learning) } \\
\hline Immediate recognition memory & 0.54 & 0.96 & -0.58 & 0.89 & 6.37 & 134 & $<0.0005$ \\
\hline Delayed recognition & 0.40 & 0.51 & -0.03 & 0.66 & 3.54 & 134 & 0.001 \\
\hline \multicolumn{8}{|l|}{ Semantic memory } \\
\hline Color naming speed (Stroop Color and Word Test, trial 1) & 0.27 & 1.00 & -1.41 & 2.65 & 5.27 & 130 & $<0.0005$ \\
\hline Verbal Category Fluency Test (animals) & 0.30 & 1.63 & -0.35 & 1.06 & 2.17 & 44 & 0.036 \\
\hline \multicolumn{8}{|l|}{ Working memory (WMS-R) } \\
\hline Digit Span backward & 0.42 & 1.06 & -0.32 & 0.97 & 3.91 & 135 & $<0.0005$ \\
\hline Visual Span backward & 0.35 & 0.93 & -0.25 & 0.89 & 3.34 & 130 & 0.001 \\
\hline \multicolumn{8}{|l|}{ Executive functions } \\
\hline Problem solving (Wisconsin Card Sorting Test, categories) & 0.45 & 0.48 & -0.26 & 1.10 & 3.73 & 128 & $<0.0005$ \\
\hline Design fluency (Ruff Figural Fluency Test) & 0.01 & 1.16 & -1.01 & 0.94 & 5.06 & 128 & $<0.0005$ \\
\hline
\end{tabular}

${ }^{a}$ All data expressed as age-adjusted z scores, except for IQ. All comparisons are two-tailed t tests.

correlations. Two-tailed tests of significance, with alpha set at 0.05 , were used throughout; however, we indicate any effects that approach significance $(\mathrm{p}<0.10)$, so that readers may consider possible type II error.

\section{Results}

\section{Two Anticholinergic Indexes: Medication Ratings, Reliability, Convergent Validity}

The relative anticholinergic activities of psychiatric medications, as estimated from either in vitro muscarinic binding or clinical ratings of peripheral anticholinergic effects, are indicated in Table 1 . The panel of 10 psychiatrists showed a high degree of agreement in their ratings of the anticholinergic effects of psychiatric medications, as assessed by the intraclass correlation coefficient (ICC $=0.88$, $\mathrm{p}<0.0001$, mixed effect model for the mean of all ratings). Using the pharmacological index of anticholinergic activity, we found that our patients were exposed to a median load of 0.28 benztropine equivalents/day (range $=0-64$ ). Using the clinical index, we found that the median load was 1.68 benztropine equivalents/day (range $=0-22$ ). Within our group of patients, these two anticholinergic indexes were highly correlated with one another $(r=0.80$, $\mathrm{p}<0.0001)$.

\section{Performance on the Neurocognitive Battery}

The neuropsychological performance of our 106 patients and 50 comparison subjects is indicated in Table 2.
The patients with schizophrenia or schizoaffective disorder performed significantly worse than the comparison subjects on every measure in this battery except Visual Span forward. To assist interpretation of findings, we performed a principal components analysis on the neurocognitive battery, which provided a moderately adequate sample for this purpose (Keyser-Meyer-Olkin index of sample adequacy $=0.76$ ). Scree-plot and factor-interpretability criteria indicated the extraction of four components, accounting for $54 \%$ of the total variability in cognitive test scores. Promax-rotated factor loadings are provided in Table 3; we interpret these factors as 1) general intelligence and attention, 2) visual attention, 3) short-term declarative memory, and 4) other.

\section{Relationship of Anticholinergic Load to Neuropsychological Performance}

Table 4 provides the Pearson correlation coefficients relating anticholinergic load to performance on the neuropsychological measures and factors. Each anticholinergic index was significantly related to inferior performance on 1) trial 1 and the sum of trials $1-5$ of the California Verbal Learning Test; 2) immediate and delayed facial memory; 3) sum of trials 1-5 on the Serial Visuospatial Learning Test; 4) copy accuracy and delayed recall on the Rey-Osterrieth Complex Figure Design; 5) Trails B, and 6) color naming speed and response control on the Stroop Color and Word Test (the color-word response control measure only 
TABLE 3. Principal Component Analysis of Neuropsychological Measures for 106 Patients With Schizophrenia or Schizoaffective Disorder ${ }^{a}$

\begin{tabular}{|c|c|c|c|c|}
\hline \multirow[b]{2}{*}{ Neuropsychological Measure } & \multicolumn{4}{|c|}{ Factor Loading } \\
\hline & $\begin{array}{c}\text { General } \\
\text { Intelligence/Attention }\end{array}$ & $\begin{array}{c}\text { Visual } \\
\text { Attention }\end{array}$ & $\begin{array}{c}\text { Short-Term } \\
\text { Declarative Memory }\end{array}$ & Other \\
\hline Intelligence (WAIS-R IQ) & $0.72^{\mathrm{b}}$ & 0.48 & 0.36 & \\
\hline Trails B & $0.72^{\mathrm{b}}$ & $0.63^{\mathrm{b}}$ & 0.40 & \\
\hline Visual Span forward (WMS-R) & $0.64^{b}$ & & & \\
\hline Digit Span backward (WMS-R) & $0.63^{\mathrm{b}}$ & & & \\
\hline Design fluency (Ruff Figural Fluency Test) & $0.60^{\mathrm{b}}$ & $0.55^{b}$ & & 0.28 \\
\hline Figure copy (Rey-Osterrieth Complex Figure Design) & $0.58^{\mathrm{b}}$ & & 0.46 & \\
\hline Digit Span forward (WMS-R) & $0.54^{b}$ & 0.35 & & \\
\hline Visual Span backward (WMS-R) & $0.53^{b}$ & 0.43 & & \\
\hline Problem solving (Wisconsin Card Sorting Test, categories) & $0.52^{\mathrm{b}}$ & & & \\
\hline Stroop Color and Word Test, color-word & 0.30 & $0.80^{\mathrm{b}}$ & & \\
\hline Color naming speed (Stroop Color and Word Test, trial 1) & & $0.78^{\mathrm{b}}$ & 0.28 & \\
\hline Trails A & $0.54^{b}$ & $0.71^{b}$ & 0.29 & 0.26 \\
\hline \multicolumn{5}{|l|}{ Verbal recall } \\
\hline Serial (California Verbal Learning Test, sum of trials 1-5) & 0.31 & & $0.83^{b}$ & \\
\hline Initial (California Verbal Learning Test, trial 1) & 0.32 & 0.33 & $0.82^{b}$ & \\
\hline \multicolumn{5}{|l|}{ Facial memory } \\
\hline Delayed recognition (Test of Memory and Learning) & & $0.51^{b}$ & $0.63^{b}$ & \\
\hline Immediate recognition (Test of Memory and Learning) & & $0.59^{\mathrm{b}}$ & $0.60^{\mathrm{b}}$ & \\
\hline Figure recall (Rey-Osterrieth Complex Figure Design) & 0.43 & & $0.60^{\mathrm{b}}$ & 0.49 \\
\hline \multicolumn{5}{|l|}{ Visuospatial recall } \\
\hline Initial (Serial Visuospatial Learning Test, trial 1) & & & & 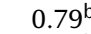 \\
\hline Serial (Serial Visuospatial Learning Test, sum of trials 1-5) & 0.26 & & 0.29 & $0.78^{\mathrm{b}}$ \\
\hline Verbal Category Fluency Test (animals) & 0.27 & 0.47 & 0.25 & 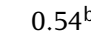 \\
\hline
\end{tabular}

a Structure matrix (item-factor correlations), following oblique rotation (Promax). All loadings $>0.03$ are shown.

${ }^{\mathrm{b}}$ Factor loading $>0.50$.

approached significant correlation with the pharmacological index). The remaining tasks of the neuropsychological battery were not associated with anticholinergic load when calculated with either method.

In terms of the neurocognitive factors, short-term declarative memory was significantly related to both anticholinergic indexes; in addition, visual attention was significantly related to the clinical anticholinergic index but not to the pharmacological index.

A chi-square homogeneity test comparing the pattern of correlations of the two anticholinergic indexes (42) found no significant difference between the correlations of the indexes with the 20 cognitive measures $\left(\chi^{2}=14.5, \mathrm{df}=20, \mathrm{p}=\right.$ $0.20)$ or with the four cognitive factors $\left(\chi^{2}=3.20, \mathrm{df}=4, \mathrm{p}=\right.$ $0.47)$.

To assess the possible role of symptom severity in mediating the effects found, we conducted partial correlation analyses on all significant correlations between anticholinergic load and neuropsychological performance, controlling for GAF score and for total scores on the extended version of the Positive and Negative Symptom Scale. This had no significant effect on any correlation (all partial correlations were within $\mathrm{r}=0.03$ of their zero-order correlations).

To assess whether the hypothesized effects of anticholinergic load on divided attention and declarative memory were independent of each other, we calculated partial correlations between anticholinergic load and Trails B performance, controlling for performance on the California Verbal Learning Test. The association between anticholinergic load and Trails B performance diminished slightly but remained significant after we controlled for ei- ther of the two California Verbal Learning Test scores ( $\mathrm{r}=$ $0.25, \mathrm{p}=0.02$ ).

To assess whether the associations between anticholinergic load and neuropsychological performance were strongest for the patients' most impaired neurocognitive functions, we calculated the correlation between 1) Pearson's correlation coefficient between anticholinergic load and neurocognitive performance and 2) the t value from the $t$ test comparing patients and control subjects on the neurocognitive battery. This secondary correlation was $\mathrm{r}=$ -0.59 , $p=0.006$, when the pharmacological index was used; it was $\mathrm{r}=-0.49, \mathrm{p}=0.03$, when the clinical index was used. These negative correlations indicate that the association of anticholinergic load with neurocognitive impairment is strongest where the performance of the schizophrenia group is closest to normal.

\section{Hypothetical Effect of Medication Dose Change on Memory Performance}

Finally, we estimated the expected impairment in episodic memory performance as the benztropine mesylate dose was escalated within the range routinely used in psychiatric treatment. We regressed patients' scores on the California Verbal Learning Test (sum of trials 1-5) on anticholinergic load (using the pharmacological index) after previously entering the total score on the extended version of the Positive and Negative Symptom Scale to control for current symptom severity, and the score on the WAIS-R Picture Completion and Information to control for IQ, excluding attention-dependent WAIS-R subtests. We then estimated the decrement in California Verbal 
TABLE 4. Correlations Between Anticholinergic Load and Neuropsychological Measures for 106 Patients With Schizophrenia or Schizoaffective Disorder ${ }^{\mathrm{a}}$

\begin{tabular}{|c|c|c|}
\hline \multirow[b]{2}{*}{ Neuropsychological Measure } & \multicolumn{2}{|l|}{$\mathrm{r}^{\mathrm{b}}$} \\
\hline & $\begin{array}{l}\text { Pharmacological Anticholinergic } \\
\text { Index }\end{array}$ & $\begin{array}{l}\text { Clinical Anticholinergic } \\
\text { Index }\end{array}$ \\
\hline Intelligence: full-scale IQ (WAIS-R) & -0.09 & -0.15 \\
\hline \multicolumn{3}{|l|}{ Simple attention } \\
\hline Digit Span forward (WMS-R) & -0.12 & -0.17 \\
\hline Visual Span forward (WMS-R) & 0.02 & -0.05 \\
\hline Visuomotor search (Trails A) & -0.14 & -0.18 \\
\hline \multicolumn{3}{|l|}{ Complex attention } \\
\hline Divided attention (Trails B) & $-0.32^{* *}$ & $-0.27^{* *}$ \\
\hline Response control (Stroop Color and Word Test, color-word) & -0.17 & $-0.30^{* *}$ \\
\hline Copy accuracy (Rey-Osterreith Complex Figure Design) & $-0.26^{*}$ & $-0.21^{*}$ \\
\hline \multicolumn{3}{|l|}{ Short-term memory } \\
\hline \multicolumn{3}{|l|}{ Verbal learning and recall } \\
\hline Initial recall (California Verbal Learning Test, trial 1) & $-0.29 * *$ & $-0.33^{* *}$ \\
\hline Serial recall (California Verbal Learning Test, sum of trials 1-5) & $-0.32^{* *}$ & $-0.29 * *$ \\
\hline \multicolumn{3}{|l|}{ Visuospatial learning and recall } \\
\hline Initial recall (Serial Visuospatial Learning Test, trial 1) & -0.10 & -0.17 \\
\hline Serial recall (Serial Visuospatial Learning Test, sum of trials 1-5) & $-0.24 *$ & $-0.26^{*}$ \\
\hline Delayed recall (Rey-Osterrieth Complex Figure Design) & $-0.24^{*}$ & $-0.24 *$ \\
\hline \multicolumn{3}{|l|}{ Facial learning and recall (Test of Memory and Learning) } \\
\hline Immediate recognition memory & $-0.22^{*}$ & $-0.24 *$ \\
\hline Delayed recognition & $-0.24^{*}$ & $-0.28^{* *}$ \\
\hline \multicolumn{3}{|l|}{ Semantic memory } \\
\hline Color naming speed (Stroop Color and Word Test, trial 1) & $-0.24^{*}$ & $-0.34 * *$ \\
\hline Verbal category fluency test (animals) & 0.00 & -0.10 \\
\hline \multicolumn{3}{|l|}{ Working memory } \\
\hline Digit Span backward (WMS-R) & 0.05 & 0.04 \\
\hline Visual Span backward (WMS-R) & -0.09 & -0.11 \\
\hline \multicolumn{3}{|l|}{ Executive functions } \\
\hline Problem solving (Wisconsin Card Sorting Test, categories) & -0.18 & -0.13 \\
\hline Design fluency (Ruff Figural Fluency Test) & -0.08 & -0.09 \\
\hline \multicolumn{3}{|l|}{ Factor scores } \\
\hline General intelligence/attention & -0.15 & -0.16 \\
\hline Visual attention & -0.14 & $-0.24^{*}$ \\
\hline Declarative memory & $-0.35^{* *}$ & $-0.35^{* *}$ \\
\hline Other & -0.14 & -0.19 \\
\hline
\end{tabular}

${ }^{a}$ All neuropsychological measures were corrected for age and sex and coded so that negative values correspond to worse performance. See Table 3 for derivation of neuropsychological factors by principal components analysis.

${ }^{\mathrm{b}}$ Pearson correlations with two-tailed significance levels.

$* \mathrm{p}<0.05 . \quad * * \mathrm{p}<0.01$.

Learning Test performance resulting from a hypothetical benztropine mesylate dose change from 0.5 to $4 \mathrm{mg} /$ day. This resulted in a decrement of 1.73 standard deviations in California Verbal Learning Test performance.

\section{Discussion}

\section{Summary of Findings}

In this study, we assessed the performance on a brief neuropsychological battery of 106 clinically stable outpatients with schizophrenia treated with a range of psychotropic medications. We compared two methods of calculating anticholinergic load in order to 1) assess each method's utility in estimating the contribution of anticholinergic activity to neuropsychological impairment, 2) identify the pattern of neuropsychological impairment associated with anticholinergic effects, and 3) determine the magnitude of these effects. We found that the clinical index, derived from expert clinicians' ratings, had high reliability (agreement among raters) and validity (correlation with the pharmacological index). Furthermore, the clinical anticholinergic index and the pharmacological index show similar patterns of associations with neuropsychological impairment. Anticholinergic load accounted for approximately $10 \%$ of the variance in measures of declarative memory function and divided attention. In addition, our data suggest that raising the anticholinergic load from 0.5 to $4 \mathrm{mg} /$ day of pharmacological benztropine mesylate equivalents results in a decrement of 1.7 standard deviations in memory performance. No effect of anticholinergic load was evident on measures of intelligence, simple attention, working memory, executive function, verbal and visual conceptual fluency, or motor speed.

\section{Anticholinergic Effects on Cognition in Schizophrenia}

These results are consistent with the small literature addressing anticholinergic effects on cognition in the pharmacological treatment of schizophrenia. Patients with schizophrenia exhibit deficits in verbal recall associated with anticholinergic loads conferred by their psychotropic medication $(4,6,7,10,11)$. Withdrawal from anticholin- 
ergics has been shown to improve overall scores on the WMS-R in subjects with schizophrenia (5), and switching to amantadine has been shown to improve scores on the Rey Auditory-Verbal Learning Test (9) and Buschke's Selective Reminding Test (8). In addition, Tracy et al. (11) found serum anticholinergic loads to correlate with Stroop Color and Word Test interference effects. Another study (43) found no association between serum anticholinergic load and Mini-Mental Status Examination total scores among subjects with schizophrenia who were treated with either clozapine or risperidone. This is consistent with our finding that anticholinergic load is not associated with general cognitive impairment.

To our knowledge, there are no previous reports assessing the effects of anticholinergic medications in subjects with schizophrenia on a complex task such as Trails B. This task involves several domains of cognitive function, including simple visual processing and visuospatial attention, as well as executive functions such as working memory, divided attention, visuomotor sequencing, and implementation of behavioral strategies. We cannot determine on the basis of these data which of these cognitive functions may be associated with anticholinergic load. Nevertheless, visuospatial attention and divided attention appear to be mediated, at least in part, by the cholinergic projections from the medial septum to the hippocampus and from the basal nucleus of Meynert to widespread cortical targets, respectively (12). The reported effects of diffuse cortical cholinergic depletion on visual or divided attention in rodents are consistent with our finding of impairment on Trails B performance in association with systemic anticholinergic loads. The Trail Making Test, therefore, appears to be sensitive to cholinergic antagonism in one or two of the three ascending forebrain cholinergic subsystems.

\section{Limitations of the Study}

It is important to note that this study was not designed in a prospective, randomized, or controlled manner. The use of correlational analyses in the post hoc analysis confers the power to indicate associations but cannot identify causal relationships between psychotropic medication use and cognitive function. One possible explanation for the hypothesized associations is that higher anticholinergic loads in this group of patients reflect a more severe degree of psychopathology, which would require higher doses of both neuroleptic and anticholinergic medication, and that the degree of pathology per se mediates this association. However, anticholinergic loads in these patients were not associated with other cognitive deficits that are typically observed in association with more severe pathology, such as those measured by the Wisconsin Card Sorting Test or other measures of executive function (44). In addition, controlling for standard measures of either symptom severity (by using an extended version of the
Positive and Negative Symptom Scale), or global function (GAF scores) had no impact on the associations observed.

Furthermore, a secondary correlation analysis indicated that the association of anticholinergic load with neurocognitive impairment was strongest when the performance of the patients with schizophrenia was closest to normal. We conclude, therefore, that the association of anticholinergic load with neurocognitive impairment is not merely a reflection of illness severity or underlying cognitive impairment in this group of subjects.

It should also be noted that our patients were exposed to a relatively high anticholinergic load, on average. This load generally exceeds the dose of antiparkinsonian medication clinically indicated for the prophylaxis/treatment of extrapyramidal symptoms associated with neuroleptic treatment. However, in routine clinical practice, the intrinsic anticholinergic activity of psychiatric medications (such as tricyclic antidepressants, paroxetine, and olanzapine) is often not considered when adding an antiparkinsonian medication to the regimen. Therefore, the effective anticholinergic load that many patients are exposed to probably exceeds the level recognized by many clinicians, as Tune et al. (45) found for medication regimens in geriatric medicine.

It is possible that our 10 psychiatrists were significantly biased by a previous knowledge of relative in vitro receptor binding affinities of the different medications. However, there was some variation in the clinical potencies assigned to each medication by the different clinician raters. It also seems likely that the degree of in vitro cholinergic receptor antagonism exhibited by medications such as olanzapine is not well recognized by clinicians. Therefore, we believe that the consistency seen between the two methods of calculating anticholinergic load is physiologically and clinically significant.

In addition, we asked our panel to rate the medications' clinical anticholinergic activity on the basis of three measures of peripheral anticholinergic effects. There is no accepted measure of central cholinergic function as a basis for assessing clinical anticholinergic effects. Nevertheless, all of these medications achieve significant penetration of the brain, which is a sine qua non of psychotropic activity. Although there are some differences in muscarinic receptor subtype distribution between the brain and the periphery $(46,47)$, it appears unlikely that a significant fraction of the present medications would show dissociation between peripheral and central anticholinergic effects.

\section{Conclusions}

Indexes of anticholinergic activity derived from either in vitro pharmacological or clinical measures show utility for estimating the cognitive effects of prescribed medications on patients with schizophrenia. These indexes represent a relatively easy, reliable, and inexpensive alternative to laboratory determinations of serum anticholinergic activity when the latter are unavailable. In patients with schizo- 
phrenia, central cholinergic antagonism is associated with selective impairments of memory and recall as well as certain complex attentional functions. This is quite consistent with animal research demonstrating roles of forebrain ascending cholinergic subsystems in memory and divided attention. Routine increases in anticholinergic medication may impair verbal memory by as much as 1.7 standard deviations. This effect cannot account fully for the memory impairments seen in schizophrenia, which often exceeds two to three standard deviations. Nonetheless, the anticholinergic load of common medication regimens may have a significant clinical impact—sufficient to shift memory performance, for example, from the normal to the impaired range (50th to fifth percentile). Likewise, this study demonstrates that future research on the cognitive functioning of psychiatric patients must account for the anticholinergic effects of medications on subjects' performance.

Received Oct. 5, 2001; revisions received Oct. 8, 2002, and July 1 , 2003; accepted July 10, 2003. From the Department of Psychiatry, Mount Sinai School of Medicine and VA Medical Center, Bronx, N.Y.; and the Department of Psychiatry, University of California and VA Medical Center, San Francisco. Address reprint requests to Dr. Vinogradov, Outpatient Mental Health, 116C, DVA Medical Center, 4150 Clement St., San Francisco, CA 94121; sophia@itsa.ucsf.edu (e-mail).

Supported in part by a VA Research Fellowship in the Clinical Neurosciences (Dr. Minzenberg) and by NIMH grant 52906-01A1 (Dr. Vinogradov).

\section{References}

1. Spohn HE, Strauss ME: Relation of neuroleptic and anticholinergic medication to cognitive functions in schizophrenia. J Abnorm Psychol 1989; 98:367-380

2. Green MF: What are the functional consequences of neurocognitive deficits in schizophrenia? Am J Psychiatry 1996; 153: 321-330

3. Green MF, Kern RS, Braff DL, Mintz J: Neurocognitive deficits and functional outcome in schizophrenia: are we measuring the "right stuff"? Schizophr Bull 2000; 26:119-136

4. Tune LE, Strauss ME, Lew MF, Breitlinger E, Coyle JT: Serum levels of anticholinergic drugs and impaired recent memory in chronic schizophrenic patients. Am J Psychiatry 1982; 139: 1460-1462

5. Baker LA, Cheng LY, Amara IB: The withdrawal of benztropine mesylate in chronic schizophrenic patients. Br J Psychiatry 1983; 143:584-590

6. Calev A: Recall and recognition in mildly disturbed schizophrenics: the use of matched tasks. Psychol Med 1984; 14 : 425-429

7. Perlick D, Stastny P, Katz I, Mayer M, Mattis S: Memory deficits and anticholinergic levels in chronic schizophrenia. Am J Psychiatry 1986; 143:230-232

8. Hitri A, Craft RB, Fallon J, Sethi R, Sinha D: Serum neuroleptic and anticholinergic activity in relationship to cognitive toxicity of antiparkinson agents in schizophrenic patients. Psychopharmacol Bull 1987; 23:33-37

9. Fayen M, Goldman MB, Moulthrop MA, Luchins DJ: Differential memory function with dopaminergic versus anticholinergic treatment of drug-induced extrapyramidal symptoms. Am J Psychiatry 1988; 145:483-486
10. Strauss M, Reynolds K, Jayaram G, Tune L: Effects of anticholinergic medication on memory in schizophrenia. Schizophr Res 1990; 3:127-129

11. Tracy JI, Monaco C, Giovannetti T, Abraham G, Josiassen RC: Anticholinergicity and cognitive processing in chronic schizophrenia. Biol Psychol 2001; 56:1-22

12. Everitt BJ, Robbins TW: Central cholinergic systems and cognition. Annu Rev Psychol 1997; 48:649-684

13. Sarter M, Bruno JP: Cognitive functions of cortical acetylcholine: toward a unifying hypothesis. Brain Res Rev 1997; 23:2846

14. Turchi J, Sarter M: Cortical acetylcholine and processing capacity: effects of cortical cholinergic deafferentation on crossmodal divided attention in rats. Cogn Brain Res 1997; 6:147158

15. O'Neill J, Fitten LJ, Siembieda DW, Crawford KC, Halgren E, Fisher A, Refai D: Divided attention-enhancing effects of AF102B and THA in aging monkeys. Psychopharmacology (Berl) 1999; 143:123-130

16. Waite JJ, Wardlow ML, Power A: Deficit in selective and divided attention associated with cholinergic basal forebrain immunotoxic lesion produced by 192-saporin; motoric/sensory deficit associated with Purkinje cell immunotoxic lesion produced by OX7-saporin. Neurobiol Learn Mem 1999; 71:325-352

17. Sarter M, Givens B, Bruno JP: The cognitive neuroscience of sustained attention: where top-down meets bottom-up. Brain Res Rev 2001; 35:146-160

18. Cooper JR, Bloom FE, Roth RH: The Biochemical Basis of Neuropharmacology, 7th ed. New York, Oxford University Press, 1996

19. Tune L, Coyle JT: Serum levels of anticholinergic drugs in treatment of acute extrapyramidal side effects. Arch Gen Psychiatry 1980; 37:293-297

20. Kremen WS, Seidman LJ, Faraone SV, Pepple JR, Lyons MJ, Tsuang MT: The "3 Rs" and neuropsychological function in schizophrenia: an empirical test of the matching fallacy. Neuropsychology 1996; 10:22-31

21. Kay SR, Opler LA, Fiszbein A: Positive and Negative Syndrome Scale (PANSS) Rating Manual. San Rafael, Calif, Social and Behavioral Sciences Documents, 1987

22. Andreasen NC, Flaum M, Arndt S: The Comprehensive Assessment of Symptoms and History (CASH): an instrument for assessing diagnosis and psychopathology. Arch Gen Psychiatry 1992; 49:615-623

23. Wechsler D: Manual for the Wechsler Adult Intelligence ScaleRevised. New York, Psychological Corp, 1981

24. Reitan RM, Wolfson D: The Halstead-Reitan Neuropsychological Test Battery. Tucson, Ariz, Neuropsychology Press, 1985

25. Wechsler D: A standardized memory scale for clinical use. J Psychol 1945; 19:87-95

26. Nelson HE: A modified card sorting test sensitive to frontal lobe defects. Cortex 1976; 12:313-324

27. Regard M: Cognitive Rigidity and Flexibility: A Neuropsychological Study (doctoral dissertation). Victoria, BC, University of Victoria, Department of Psychology, 1981

28. Denman SB: Denman Neuropsychology Memory Scale. Charleston, SC, SB Denman, 1987

29. Delis DC, Kramer JH, Kaplan E, Ober BA: The California Verbal Learning Test Manual. New York, Psychological Corp, 1987

30. Reynolds CR, Bigler ED: Test of Memory and Learning. Austin, Tex, PRO-ED, 1994

31. Tombaugh TN, Kozak J, Rees L: Normative data for the Controlled Oral Word Association Test (1996), in A Compendium of Neuropsychological Tests. Edited by Spreen O, Strauss E. New York, Oxford University Press, 1998, pp 453-457

32. Ruff R: Ruff Figural Fluency Test. San Diego, Calif, Neuropsychological Resources, 1998 
33. Reitan RM: Manual for the Administration of Neuropsychological Test Batteries for Adults and Children. Indianapolis, RM Reitan, 1969

34. Cusack B, Nelson A, Richelson E: Binding of antidepressants to human brain receptors: focus on newer generation compounds. Psychopharmacology (Berl) 1994; 114:559-565

35. El-Fakahany E, Richelson E: Antagonism by antidepressants of muscarinic acetylcholine receptors of human brain. Br J Pharmacol 1983; 78:97-102

36. Richelson E: Neuroleptic affinities for human brain receptors and their use in predicting adverse effects. J Clin Psychiatry 1984; 45:331-336

37. Richelson E, Nelson A: Antagonism by antidepressants of neurotransmitter receptors of normal human brain tissue in vitro. J Pharmacol Exp Ther 1984; 230:94-102

38. Saller CF, Salama Al: Seroquel: biochemical profile of a potential atypical antipsychotic. Psychopharmacology (Berl) 1993; 112:285-292

39. Schotte A, Janssen PFM, Gommeren W, Luyten WHML, Van Gompel P, Lesage AS, De Loore K, Leysen JE: Risperidone compared with new and reference antipsychotic drugs: in vitro and in vivo receptor binding. Psychopharmacology (Berl) 1996; 124:57-73

40. Snyder S, Greenberg D, Yamamura H: Antischizophrenic drugs and brain cholinergic receptors: affinity for muscarinic sites predicts extrapyramidal effects. Arch Gen Psychiatry 1974; 31: 58-61

41. Snyder S, Yamamura H: Antidepressants and the muscarinic acetylcholine receptor. Arch Gen Psychiatry 1977; 34:236-239

42. Meng X-L, Rosenthal R, Rubin DB: Comparing correlated correlation coefficients. Psychol Bull 1992; 111:172-175

43. Tracy JI, Monaco CA, Abraham G, Josiassen RC, Pollock BG: Relation of serum anticholinergicity to cognitive status in schizophrenia patients taking clozapine or risperidone. J Clin Psychiatry 1998; 59:184-188

44. Cornblatt BA, Green MF, Walker EF: Schizophrenia: etiology and neurocognition, in Oxford Textbook of Psychopathology. Edited by Millon T, Blarney PH, Davis RD. New York, Oxford University Press, 1999, p 292

45. Tune L, Carr S, Hoag E, Cooper T: Anticholinergic effects of drugs commonly prescribed for the elderly: potential means for assessing risk of delirium. Am J Psychiatry 1992; 149:13931394

46. Brann MR, Ellis J, Jorgenson H, Hill-Eubanks D, Jones SV: Muscarinic acetylcholine receptor subtypes: localization and structure/function. Prog Brain Res 1993; 98:121-127

47. Levey Al: Immunological localization of m1-m5 muscarinic acetylcholine receptors in peripheral tissues and brain. Life Sci 1993; 52:441-448 